\title{
L'AUTOFINANCEMENT DES COOPÉRATIVES DE TRAVAILLEURS ET LA THÉORIE ÉCONOMIQUE (*)
}

\author{
par \\ Jacques DEFOURNY. \\ Chargé de recherche au CIRIEC. \\ Assistant à l'Université de Liège
}

\section{INTRODUCTION}

\section{Le contexte de cette recherche}

On constate depuis quelques années un net regain d'intérêt pour la coopération. en particulier pour les coopératives de travailleurs. En France, plus de 700 sociétés coopératives ouvrières de production (SCOP) ont vu le jour au cours des trois dernières années (1979-1982), ce qui a multiplié par 2.5 leur nombre dans ce pays. L'expérience italienne est encore plus étonnante : depuis 1977, chaque année a vu se créer quatre à cinq cents coopératives de production pour l'ensemble des trois grandes fédérations nationales. Elles ont engendré annuellement entre 1.500 et 1.700 emplois. Au Royaume- Uni, pays de vieille tradition coopérative, la Cooperative Production Federation, qui comptait plus de cent sociétés en 1900. les a vues disparaître les unes après les autres au point qu'elles sont moins d'une vingtaine aujourd'hui. Mais, parallèlement, d'autres mouvements sont nés au cours de la dernière décennie, notamment l'Industrial Common Ownership Movement, si bien que depuis 1976 près de 500 coopératives de travailleurs ont été mises sur pied. Enfin, aux Pays-Bas, on compte à présent plus de 400 coopératives de travailleurs alors qu'elles n'étaient que 80 il y a cinq ans.

(*) Ce texte fait partie d'une étude plus large intitulée "L Le renouveau des cooperatives de travailleurs : une analyse economique "publiée par le Centre européen "Travail et société "(Matistricht) dans sa collection "Work and Social Change". 
Divers autres pays d'Europe et d'Amérique du Nord connaissent actuellement des phénomènes semblables quoique souvent de moindre ampleur. Ainsi la Belgique a, elle aussi, vu émerger à un rythme croissant de multiples expériences alors que les mouvements coopératifs belges ne comptaient pratiquement aucune coopérative de travailleurs $\left({ }^{(}\right)$.

Le plus souvent, ce n'est pas une adhésion à la doctrine coopérative, d'ailleurs très méconnue, qui meut les créateurs de ces nouvelles entreprises, mais généralement ceux-ci trouvent dans la formule coopérative la seule alternative légale aux statuts classiques des sociétés capitalistes et, dans l'esprit qui la sous-tend, l'expression au moins partielle de leur volonté d'entreprendre et de travailler autrement.

Qu'elles soient créées ex nihilo, qu'elles soient le produit de la transformation d'une P.M.E. patronale en bonne santé ou le résultat d'une reprise par les travailleurs de leur entreprise défaillante, ces nouvelles coopératives attirent de plus en plus l'attention : par les emplois qu'elles engendrent ou souvent par le dynamisme qu'elles manifestent et par l'expérimentation sociale qu'elles représentent, elles apparaissent comme une voie possible, parmi d'autres, bien sûr, pour tenter de sortir de la crise actuelle. Cela est d'autant plus vrai que les nouvelles coopératives, en étant à la croisée de divers courants d'idées contemporaines, correspondent bien à l'esprit du temps $\left({ }^{2}\right)$.

Si le développement potentiel des nouvelles coopératives est problablement sous-estimé à l'heure actuelle, celles-ci n'en rencontrent pas moins certains obstacles qui conditionnent largement leur avenir. Parmi ces problèmes, celui du financement est certainement un des plus cruciaux. En effet. la faiblesse des fonds propres a toujours marqué et continue aujourd'hui de caractériser la plupart des coopératives de travailleurs. D'autre part, les organismes financiers traditionnels, en particulier les banques privées, prêtent difficilement à ce type d'entreprises : le manque de garanties offertes par les coopératives, la méconnaissance générale de celles-ci et la variabilité de leur capital $\left(^{3}\right)$ rendent généralement les prêteurs très réticents.

C'est pour ces raisons ainsi que pour assurer une certaine autonomie à leur mouvement que les coopératives de travailleurs dans l'un ou l'autre pays se sont dotées d'organismes financiers propres adaptés à leurs besoins. Le cas de la Caja Laboral Popular du

(') Pour une description générale de ce renozveiau coopératif, voir notamment J. DeFOURny (1982)

(2) C'est un des thèmes que nous avons développé dans l'aricle précite.

(') En Droit belge en tout cass. la coopérative est une société cormmerciale a capital variable 
complexe de Mondragon (pays basque espagnol) est sans doute le plus remarquable $\left({ }^{4}\right)$. En France, le SOCODEN-FEC (Société coopérative d'entraide - Fonds d'expansion confédéral) créé par la Confédération générale des SCOP dispose de moyens assez limités mais son action est exemplaire à bien des égards.

D'autre part, s'ils souhaitent favoriser le développement de coopératives de travailleurs, les pouvoirs publics peuvent aussi créer un organisme financier spécialisé. Ainsi, le gouvernement français, en 1938, a mis sur pied la Caisse centrale de crédit coopératif pour soutenir et renforcer le mouvement SCOP. Plus récemment, la Société de développement coopératif est née en 1977 de la reconnaissance pour le gouvernement québecois du rôle que pouvait jouer le mouvement coopératif dans le développement de la Province $\left({ }^{5}\right)$.

En Belgique, la Fédération des coopératives socialistes réclame depuis un certain temps déjà la création de fonds régionaux pour promouvoir le développement coopératif, en particulier celui des nouvelles coopératives. Les organisations qui tentent de fédérer ces dernières expriment le même vœu. Comme d'un autre côté certains ministères portent un intérêt croissant à la création d'emplois par le biais des nouvelles coopératives, on peut penser que ce projet a des chances d'aboutir.

Dans ce contexte et vu qu'il existe une véritable théorie économique de l'entreprise autogérée pouvant s'appliquer aux coopératives de travailleurs (voir ci-dessous), il nous a paru utile d'interroger cette théorie et de regarder si elle n'avait pas certains éléments à apporter à l'analyse et à la résolution des problèmes financiers des coopératives.

\section{Coopératives de travailleurs et autogestion}

Dans les pays où la loi reconnaît le statut de coopérative de production ou de coopérative de travailleurs, ces termes visent à signifier qu'en général les membres de la société apportent des parts de capital en vue d'exercer ensemble un travail de production (au sens large : production de biens ou services). Les travailleurs sont donc alors les principaux coopérateurs et, dans cette mesure, c'est à eux qu'appartient le pouvoir de gestion de l'entreprise, que celuici soit exercé directement ou par voie de délégation.

$\left({ }^{4}\right)$ Pour une description détaillée de la CLP. voir H. THOMAS \& C.I OOGAN (1982).

( $\left.{ }^{5}\right)$ Ces deux expériences française et québecoise ont été analysées par M.-A. SAIVE (1981). 
Dans d'autres pays, comme la Belgique par exemple, on désigne par le terme générique de nouvelles coopératives un ensemble imparfaitement défini d'entreprises créées ou sauvées collectivement par un certain nombre de personnes. Le statut juridique de coopérative est le plus souvent choisi mais ce n'est pas toujours le cas. Pour différencier ces entreprises des coopératives traditionnelles d'usagers, on les appelle aussi coopératives de travailleurs en ce sens que leur gestion, comme leur création, relèvent essentiellement d'une démarche collective des individus qui y travaillent. Elles sont donc elles aussi fondamentalement autogérées.

De ce qui précède il résulte qu'une théorie économique concernant l'entreprise autogérée s'applique sans problème majeur aux coopératives de travailleurs $\left(^{6}\right)$. Les seules réserves à faire quant à la pertinence d'une telle théorie concernent, comme dans tout modèle économique, les hypothèses simplificatrices émises au départ de' l'analyse pour caractériser les agents économiques et le contexte dans lequel ils opèrent.

\section{La théorie économique de l'autogestion}

Depuis une douzaine d'années, un nombre croissant d'économistes se sont intéressés aux problèmes de l'entreprise autogérée. I ess articles de B. Ward (1958) et de E. Domar (1966) renfermaient sans doute les principaux germes de la vaste théorie économique développée par la suite, mais c'est surtout la "General Theory of LaborManaged Market Economies " de Jaroslav Vanek, parue en 1970. qui a véritablement fait école. En effet, ce livre offrit une large analyse de l'autogestion, tant microéconomique que macroéconomique, qui put servir de base aux travaux de nombreux autres chercheurs.

Le problème de l'investissement dans l'entreprise autogeree était déjà traité par la "General Theory" (chapitre 14). Le thème du financement de cet investissement prit cependant une importance toute particulière avec un article que Vanek écrivit un an plus tard (1971). Introduisant son analyse, l'auteur affirmait textuellement : "Les arguments présentés ci-dessous me paraissent si forts qu'ils expliquent dans une large mesure l'échec historique relatif (c'est-àdire par rapport aux entreprises capitalistes classiques) des formes traditionnelles de coopératives de producteurs et d'entreprises participatives".

(1) Pour une évocation plus systématique des rapports entre autogestion et cou-

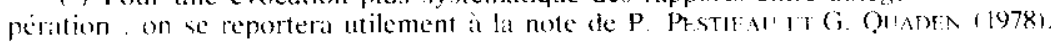


Cette étude du financement de l'entreprise autogérée a engendré de nombreux autres développements sur le plan théorique et dans une moindre mesure sur le plan empirique. Aussi le but de cette troisième partie est de faire le point sur ces diverses contributions en reprenant l'essentiel du modèle initial de Vanek et en passant en revue la littérature qui s'est placée dans son sillage. Notons cependant que la partie de la littérature consacrée spécifiquement à l'autogestion yougoslave sera évoquée mais non reprise dans le détail : parce que cette dernière relève d'un véritable système économique mis en place au niveau d'une nation. elle peut difficilement servir de référence pour des coopératives de travailleurs obligées. comme en Europe occidentale, d'opérer dans un environnement capitaliste traditionnel.

\section{I.ES QUATRE FORCES DF VANEK}

Imaginons une firme gérée démocratiquement par l'ensemble de ses travailleurs. Elle utilise du capital $\mathrm{K}$, du travail $\mathrm{L}$ fourni par ses seuls membres et des inputs variables $V$ autres que le travail. $P_{k}$ et $P_{V}$ sont les prix respectifs de $K$ et $V$. Le capital est sensé avoir une durée de vie illimitée, ce qui supprime les problèmes de dépréciation et d'amortissement.

On suppose dans un premier temps que l'entreprise opère en rendements d'échelle constants et qu'elle produit un output $X$ vendu au prix unitaire $P_{x}$. L'objectif des travailleurs est de maximiser leur revenu moyen, ce qui, formellement, se traduit par la maximisation de

$$
Y=\frac{\left(P_{x} \cdot X-P_{k} \cdot K-P_{v} \cdot V\right)}{L}
$$

Considérons le cas où l'entreprise n’a rien à payer pour le capital qu'elle utilise. Cela signifie que ses investissements sont exclusivement réalisés grâce à un autofinancement collectif (mise en réserve de bénéfices) ou individuel (capital non rémunéré et non récupérable apporté par les travailleurs lors de la fondation de l'entreprise ou de leur entrée dans celle-ci). Cette hypothèse peut aussi traduire des situations plus particulières. par exemple le cas où la propriété de l'entreprise a été donnée à la communauté nationale par voie de nationalisation et où l'Etat n'exige aucun intérêt sur le 
capital laissé en usufruit aux travailleurs $\left({ }^{7}\right)$. Sans rémunération du capital, la fonction d'objectif se réduit à

$$
\mathrm{J}=\frac{\mathrm{P}_{\mathrm{x}} \cdot \mathrm{X}-\mathrm{P}_{\mathrm{v}} \cdot \mathrm{V}}{\mathrm{L}}
$$

Sur base de cette équation on peut faire apparaitre différentes forces qui vont agir sur le comportement de l'entreprise.

1) On voit que $y$ augmente avec le produit moyen du travail $\frac{X}{L}$. Comme $\frac{X}{L}$ est inversément lié à $L$, toute réduction du nombre de travailleurs augmente $J$. Il est probable que la coopérative interdise le licenciement de ses membres mais elle aura toujours intérêt à ne pas remplacer des travailleurs qui quittent volontairement ou qui partent à la retraite, sauf si le nouveau membre apporte assez de capital pour accroître $\frac{X}{L}$.

Cette première force que Vanek appelle "première force d'autoextinction» ( first self-extinction force ») résulte du fait que le revenu par travailleur $(\mathrm{Y})$ inclut à la fois un revenu du travail et une rente du capital. Moins nombreux sont les travailleurs de l'entreprise, plus importante est la part de cette rente que chacun reçoit.

2) Si $\mathrm{L}$ décroît comme on vient de le voir, la productivité marginale du capital $\left(X_{K}\right)$ fait de même, toutes autres choses restant égales. et le rapport capital-travail s'accroît. Cela a pour effet de pousser l'entreprise à désinvestir : le rendement de certains investissements est maintenant considéré comme trop bas et l'entreprise va chercher à retrouver une productivité marginale du capital plus élevée par un désinvestissement progressif ou une consommation du capital. Notons cependant que tout rétablissement du taux de rendement désiré est seulement momentané car la tendance à réduire le nombre de membres continue de se manifester. Vanek appelle cette incitation à désinvestir "la seconde force d'auto-extinction".

Graphiquement, on peut représenter ces deux forces de la façon suivante. Pour des rendements d'échelle constants, la fonction $\frac{\mathrm{X}}{\mathrm{L}}=\mathrm{f}\left(\frac{\mathrm{K}}{\mathrm{L}}\right)$ exprimant la production par travailleur doit

(?) Une illustration parmi d'autres: dans de nombreux pays an voie de develop pement. des réformes agraires ont donné à des paysans organisés en cospératives des terres qui constituent done du capital non rémunéré 
être strictement concave (graphique 1). A n'importe quel point, la pente de la tangente à la courbe, par exemple la pente de dc au point $c$, mesure le produit marginal du capital, le segment bc mesurant le revenu du capital par travailleur, si le capital est payé à son produit marginal $\left({ }^{8}\right)$, et le segment ab mesurant le taux de salaire, si le travail est aussi payé à son produit marginal. Dans notre cas cependant, l'entreprise s'autofinance totalement et ne rémunère donc pas le capital. Puisqu'il n'y a pas de revenu du capital, les segments ab et bc n'ont plus de sens et le revenu par travailleur est équivalent à la distance ac si l'entreprise a un rapport capital-travail égal à a.

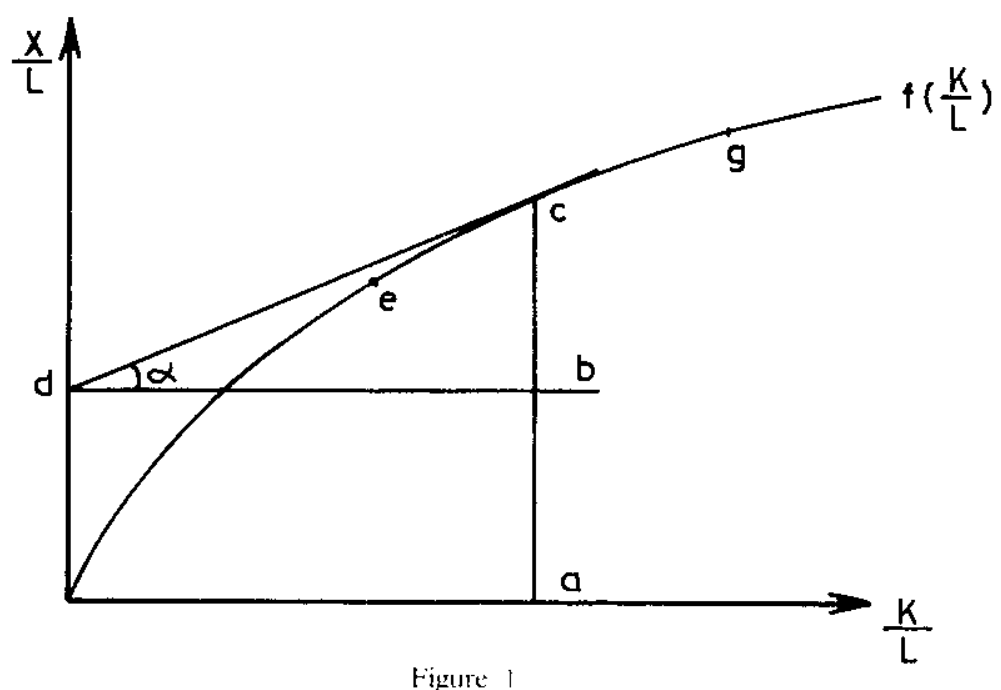

La première force d'auto-extinction apparaît très facilement : en réduisant le nombre de ses membres l'entreprise accroît $\frac{\dot{K}}{L}$ et déplace son point d'équilibre vers la droite. ce qui augmente le revenu par travailleur.

Quant à la seconde force d'auto-extinction, elle s'illustre comme suit. Supposons que les points a et c correspondent réellement à un

$\left.{ }^{*}\right)$ En effet, puisque dbc constitue un triangle rectangle, on a :

ce qui représente bien le revenu du capital par travailleur.

$$
b c=d b \cdot \operatorname{tg} \alpha-\frac{k}{1} \cdot X_{k}=\frac{k \cdot x_{K}}{1}
$$


équilibre de la préférence temporelle dans le sens ou la communauté des travailleurs n'a aucun désir d'investir ou de désinvestir $\left({ }^{9}\right)$. Comme $\mathrm{L}$ a tendance à diminuer et l'entreprise à se déplacer vers la droite de c. la productivité marginale du capital baisse (la pente de la tangente à la courbe diminue). Certains investissements n'ont plus un rendement suffisant et cela rompt l'équilibre atteint précédemment. Les travailleurs vont dès lors chercher en désinvestissant à revenir au point d'équilibre correspondant à a. avec des niveaux $\mathrm{K}$ et $\mathrm{L}$ plus bas qu'auparavant.

3) Vanek reprend en troisième lieu une force de sous-investissement ("under-investment force") mise en lumière par S. Pejovich (1969) et par E. Furubotn et S. Pejovich (1970). Selon la théorie traditionnelle de l'investissement, un individu rationnel investira pour autant que le taux de rendement $\mathrm{A}$ de l'investissement soit au moins égal à son taux de préférence temporelle $\mathbf{R}$. On parlera de sous-investissement s'il n'investit pas alors que le taux de rendement dépasse celui de sa préférence temporelle.

Cette théorie suppose implicitement que l'investisseur récupère le principal au bout de la période de l'investissement. Or. dans les entreprises autogérées, en particulier les coopératives de travailleurs, les bénéfices retenus et mis en réserves sont le plus souvent considérés comme une propriété commune. Cela signifie que dans une entreprise autogérée qui finance elle-même ses investissements aucun travailleur ne compte récupérer une part quelconque du montant investi. Par conséquent, les individus qui composent l'entreprise ne se décideront en faveur d'un investissement que si le taux de rendement $A$ dépasse $R$ d'un montant $D$ nécessaire pour compenser la non-récupération du principal. En d'autres termes le critère d'investissement s'écrit :

$$
A \geqslant R+D \text { avec } D>0
$$

Le facteur $D$ est variable et augmente au fur et à mesure que le preneur de décision se rapproche de sa retraite. On peut le vérifier de la façon suivante : si le principal de l'investissement n'est pas récupéré. la valeur présente d'une unité monétaire investie est donnée par

$$
V=A \sum_{i}^{T}(1+R) \quad i
$$

\footnotetext{
(") On suppose que tous les travatilleurs ont la meme preference temporelle $\mathrm{R}$ De façon plus explicite. dans le cas simple de deux périodes. cela signifie que chaque travacilleur comsent à épargner ou investir par exemple une unité monétaire. ê donc renonce a une partie de sal consommation courante. a condition qu'il puisse recevoir en retour l'annee suivante an moins $I+R$ enites monetaires
} 
$\mathrm{T}$ étant la période totale de l'investissement $\left({ }^{10}\right)$. Si on suppose $\mathrm{R}=$ $6 \%$, un jeune travailleur qui compte encore rester 30 ans dans une coopérative acceptera d'investir une unité monétaire si

$$
\text { A } \sum_{i=1}^{30}(1,06) \quad i \geqslant 1
$$

c'est-à-dire si $\mathrm{A} \geqslant 7,2 \%$. Dans ce cas $\mathrm{D}$ est donc égal à $1,2 \%$. Par contre par un membre de l'entreprise qui se trouve à deux ans de sa retraite, il faut que

$$
A \underset{i=1}{2}(1,06)-1 \geqslant 1
$$

c'est-à-dire $A \geqslant 54,5 \%$. Ici $\mathrm{D}$ est extrêment élevé $(48,5 \%)$. Si la structure par âge de la coopérative est stable, la tendance de celle-ci à sous-investir restera constante. Cependant on a vu qu'il existe aussi une incitation à ne pas remplacer les membres qui quittent l'entreprise. Le groupe de travailleurs tend donc à vieillir, ce qui accentue la force de sous-investissement. Mc Gregor (1977) estime même qu'une communauté de travailleurs proches de la retraite sera fortement poussée à vendre l'entreprise, c'est-à-dire à réaliser le capital accumulé, et à partager entre ses membres le produit de la vente.

4) Pour la dernière force mise en évidence par Vanek, référons-nous à nouveau au graphique 1 et supposons que la firme se situe au point $\mathrm{e}$ tandis que le niveau d'équilibre $\mathrm{A}$ * de la productivité marginale du capital correspond au point c. Au point g, cette dernière est égale au taux de préférence temporelle $\mathrm{R}$ des travailleurs. Puisqu'en e la productivité marginale du capital est supérieur à $\mathrm{A}^{*}$, les membres de la communauté auront tendance à investir et à développer la production avec un rapport $\frac{\mathrm{K}}{\mathrm{I}}$ croissant, aussi longtemps que $\frac{\mathrm{K}}{\mathrm{L}}$ restera inférieur au niveau indiqué par a sur le graphique. Dans le cadre de nos hypothèses (autofinancement et rendements d'échelle constants), c'est la seule situation où la croissance de la firme se manifeste, à condition bien sûr que le facteur travail reste constant et que la première force d'auto-extinction ne joue pas. Notons cependant, et c'est la

(10) Si le principal est récupéré. la valeur présente d'une unité monétaire investie est donnée par

$$
V=A \underset{i}{\stackrel{T}{\Sigma}(1+R)^{i}}+(1+R)^{T}
$$


force d'inemploi ( " never-employ force »), que l'entreprise n'embauchera en aucun cas de nouveaux travailleurs car cela réduirait te rapport capital-travail et donc le revenu par travailleur.

II. VERS UN MODELE DE BASE PI.US REAIISTI

Voyons à présent ce qui se passe lorsqu'on relâche certaines hypothèses du modèle décrit ci-dessus.

\section{a. L'engagement de travailleurs non membres}

Le quatrième effet pervers, celui qui résulte de la force d'inemploi, se transforme largement si, comme Mc Gregor (1977), on change dans un sens très réaliste une hypothèse du modèle. Jusqu“à présent en effet, on supposait que tous les travailleurs participent à la gestion de l'entreprise. Or on sait que dans la plupart des cas, il n'en va pas ainsi : dans les SCOP françaises par exemple, la proportion moyenne de sociétaires parmi les travailleurs est de $55 \%$. même si pour les plus jeunes coopératives ce pourcentage est souvent proche de 100 . Il n'est donc pas inutile de regarder quel genre de comportement adoptera une entreprise autogérée qui peut embaucher des travailleurs sans les admettre comme membres à part entière.

En fait, il apparaît que dans un tel cas il est toujours intéressant pour le collectif des travailleurs membres d'engager des travailleurs non membres si le salaire octroyé à ces derniers est inférieur au revenu par membre. Cela peut s'illustrer facilement sur le graphique 2 où, pour simplifier, seul le travail est considéré comme facteur variable.

Partons d'une situation où l'entreprise compte oe membres et aucun autre travailleur. Le revenu par tête $(y)$ est alors égal à $\frac{\mathrm{ef}}{\mathrm{oe}}$ qui est aussi le produit moyen du travail. Supposons à présent que des travailleurs extérieurs puissent être embauchés pour un salaire égal à la pente de la droite P2 $(\operatorname{tg} \alpha)$.

Dans ce cas, le revenu par membre sera maximisé par l'engagement de ea travailleurs : en effet, au point a, la productivité marginale du travail est égale au prix de ce dernier. Les travailleurs additionnels ajoutent un montant bd à la production alors que leur coût 
salarial global est seulement égal à $\operatorname{cd}(\operatorname{tg} \alpha$, ea $=\operatorname{tg} \alpha \cdot g c=c d)$. Le revenu par membre s'est donc accru de $\frac{b c}{o e}\left({ }^{11}\right)$.

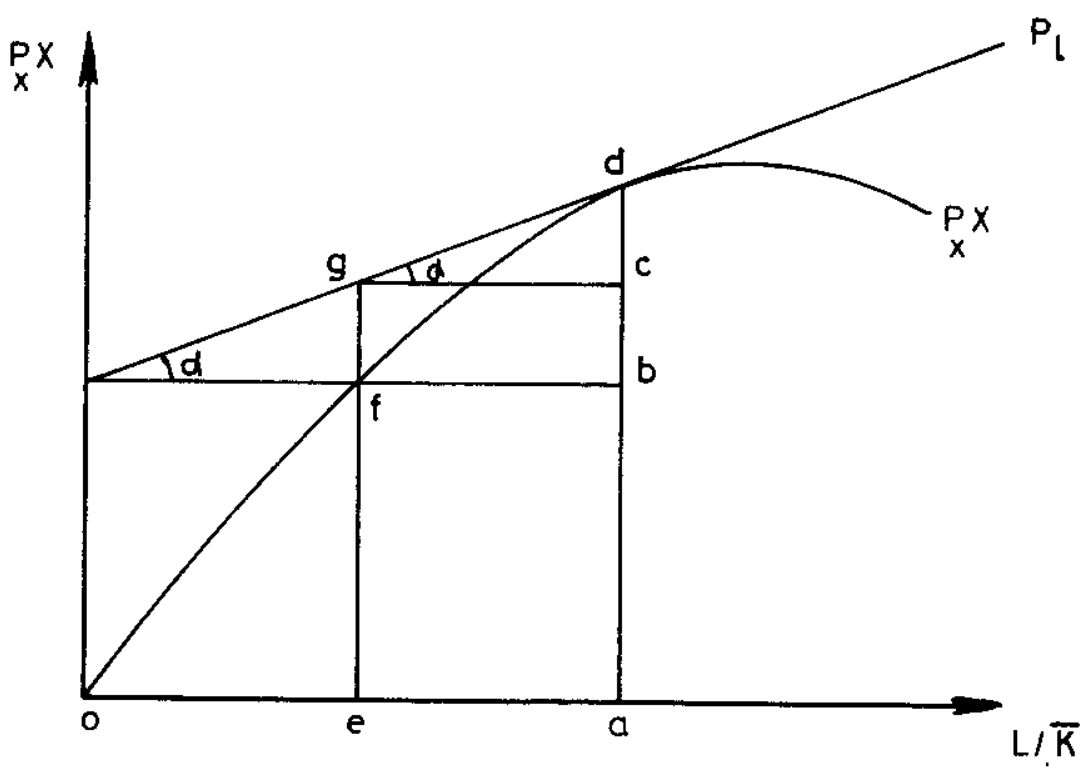

Figure 2

b. Des rendements d'échelle variables

Vanek lui-même envisage le cas où la fonction de production se caractérise par des rendements d'échelle croissants pour de faibles niveaux d'output, puis par des rendements d'échelle décroissants ou constants pour des niveaux de production supérieurs. A l'exception des coopératives de production agricole pour lesquelles l'hypothèse des rendements d'échelle constants est souvent acceptable, il s'agit là aussi d'une modification qui rend le modèle plus proche de la réalité économique.

L'analyse peut être menée à partir du graphique 3. Sur celui-ci, la courbe EE représente le lieu géométrique de l'efficience physique maximale : chaque point de EE représente une situation où, pour un rapport capital-travail donné, les productivités moyennes des deux

(11) C. BELLAS (1972) prédisait déjà lextinction de coopératives prospères par l'engagement d'un nombre croissant de salariés non membres, c'est-à-dire par la perte de leur identité coopérative. 
facteurs, $\frac{X}{K}$ et $\frac{X}{I}$, sont maximisées. C'est aussi un lieu le long duquel le théorème d'Euler se vérifie, c'est-à-dire où on a $X=X_{L} L=X_{K} K$. Vanek (1970, pp. 27-38) a montré qu'une entreprise aútogérée financée de l'extérieur et opérant dans des conditions de concurrence parfaite se situera toujours dans le long terme sur la courbe EE ou au-delà de celle-ci (de la même manière qu'une firme capitaliste opérant dans des conditions identiques).

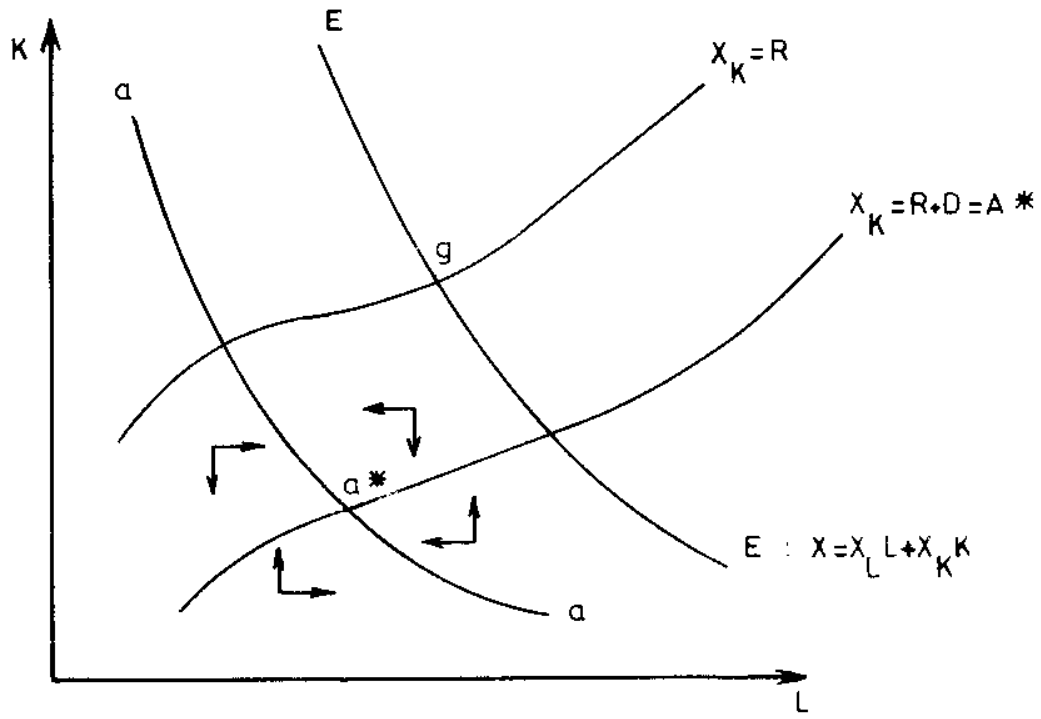

Figure 3

Sur la courbe EE, la technologie est "localement " soumise à des rendements d'échelle constants. A gauche et en dessous de la courbe les rendements sont croissants tandis qu'à sa droite et audessus ils sont soit décroissants soit constants.

Les quatre forces mises en lumière par Vanek pour le cas des rendements d'échelle constants agissent donc localement le long de la courbe EE. C'est pourquoi un équilibre ne pourra y exister pour l'entreprise autogérée qui se base sur un autofinancement collectif. En réalité, l'équilibre de celle-ci devra se situer quelque part sur la courbe aa qui est le lieu le long duquel, pour des niveaux donnés de capital, les productivités moyenne et marginale du travail sont égales. Sur ce lieu, le produit moyen du travail $\frac{\mathrm{X}}{\mathrm{l}}$ est donc à son 
niveau maximum. Or c'est bien là l'objectif que poursuit une firme autogérée autofinancée puisque, aucun paiement ne devant être fait pour le capital utilisé, la maximisation du produit moyen du travail entraîne la maximisation du revenu par travailleur. Malheureusement, le lieu aa se trouve dans la zone des rendements d'échelle croissants et est donc techniquement inefficient. On peut vérifier que la dynamique qui amène l'entreprise à opérer sur la courbe aa est bien analogue aux diverses forces décrites plus haut. En particulier, l'effet résultant de la force de sous-investissement peut s'illustrer comme suit. Avec une épargne collective irrécupérable, on a vu que le produit marginal d'équilibre du capital doit se placer bien au-dessus du taux de préférence temporelle $R$, à un niveau $A^{*}=R+D$. Cela définit un nouveau lieu le long duquel $X_{K}=A^{*}$ et nous avons un équilibre pour notre firme autogérée autofinancée au point $\mathrm{a}^{*}$, à l'intersection de aa et $\mathrm{X}_{\mathrm{K}}=\mathrm{A}^{*}$.

Les forces d'ajustement dynamique pour n'importe quel point éloigné de l'équilibre $\mathrm{a}^{*}$ sont indiquées sur le graphique 3 . A la droite (respectivement à la gauche) de aa, l'emploi diminuera (resp. augmentera) de façon à accroître le revenu par travailleur tandis qu'en dessous (resp. au dessus) de $\mathrm{X}_{\mathrm{k}}=\mathrm{A} *$, il y a accumulation (resp. consommation du capital) conformément à la préférence temporelle des travailleurs. En conséquence, $a^{*}$ est un point d'équilibre stable.

A contrario, avec un financement d'origine externe et un coût du capital $R$ reflétant sa rareté. l'équilibre d'une entreprise autogérée devra se situer sur la courbe $X_{K}=R$. Comme d'autre part, le revenu par travailleur sera maximisé en n'importe quel point du lieu EE d'efficience physique maximale, l'équilibre d'une entreprise autogérée financée de l'extérieur se trouvera à l'intersection de ces deux dernières courbes, c'est-à-dire en $\mathrm{g}$.

En résumé, on peut conclure de ce qui précède que, par rapport à une situation de financement externe avec un coût du capital égal au taux de préférence $R$, l'autofinancement conduit la firme autogérée à une production inefficiente de quantités relativement faibles, et à un sous-investissement.

\section{c. Un autofinancement seulement partiel}

Enfin, par souci de réalisme. on peut aussi relâcher l'hypothèse d'un financement interne collectif à $100 \%$ et permettre un certain degré de financement externe ou de financement interne par apports individuels récupérables. En fait, ces nouvelles conditions diminuent les effets pervers mis en évidence par le modèle mais ne les éliminent pas. Pour le comprendre, il suffit de voir que si l'entreprise autogérée doit rémunérer une partie de son capital au taux d'intérêt du marché. le lieu des revenus maximum par travailleur va 
se déplacer de aa vers la droite sur le graphique 3. Il ne se confond donc plus avec le lieu des productivités moyennes maximum du travail. Cependant, il n’ira pas jusqu’à se confondre avec le lieu EE. ce qui ne serait possible quavec un financement totalement externe.

D'autre part, si la communauté des travailleurs peut s'attendre à ce qu'une proportion donnée de tout investissement soit financée de l'extérieur à un taux $R$, cela réduit le montant de compensation $D$ et rapproche $A *$ de $R$. Sur le graphique 3, le lieu correspondant à $X_{K}=A *$ remonte mais pas jusqu'à $X_{K}=R$, et la solution d'équilibre de l'entreprise se déplace vers l'intersection des deux nouvelles courbes.

\section{d. Une approche dynamicue}

Alors que les travaux de Ward (1958), Domar (1966) et Vanek (1970 et 1971) reposent sur des modèles statiques, la prise en considération de la dimension "temps" permet d'introduire dans l'analyse une réalité déjà évoquée avec la "troisième force " de Vanek mais pas encore formellement intégrée : en renonçant. en la période $t=1$. à une partie des revenus qu'ils s'octroieraient s'ils visaient seulement à maximiser leurs salaires courants. les travailleurs peuvent financer des investissements qui, par le biais d'une productivité accrue. engendreront des revenus plus élevés dans les périodes $\mathrm{t}=2.3, \ldots \mathrm{T}$.

Envisageant une firme dont la technologie se caractérise par des rendements d'échelle croissants, A.B. Atkinson (1973) reprend simplement mais en la dynamisant l'hypothèse de maximisation du revenu par travailleur. En d'autres termes, l'objectif de la firme devient la maximisation de la valeur présente (obtenue par une formule d'actualisation) du revenu par tête.

La partic essentielle du travail d'Atkinson consiste en une analyse pour laquelle il suppose un financement totalement externe. Sur cette base, il étudie les décisions d'investissement et de prix d'une entreprise autogérée qui maximise la valeur présente du revenu par travailleur ("labor-managed firm ") mais aussi d'une grande firme moderne autogérée dont l'objectif, dans le chef des dirigeants élus, serait plutôt de rendre maximum la croissance de la firme sous la contrainte de maintenir le revenu par travailleur audessus d'un certain niveau donné ( managerial labor-owned firm »).

C'est seulement en fin d'analyse que l'auteur introduit la possibilité d'un financement interne collectif par la mise en réserve de profits. Cela se traduit par un paramètre $\lambda$ qui représente la proportion du capital total financée par emprunt à l'extérieur de l'entre- 
prise, l'expression $1-\lambda$ donnant dès lors la part d'autofinancement. Dans le cas de la "labor-managed firm ", Atkinson arrive au résultat suivant : plus le financement est interne, c'est-à-dire plus $1+\lambda$ est élevé, plus l'investissement et le taux de croissance de l'entreprise seront faibles. Il montre de surcroît que cette conclusion reste valable quels que soient les taux d'intérêt sur les capitaux que la firme peut emprunter.

L'approche dynamique que propose Furubotn (1971) s'inscrit quant à elle dans une hypothèse de maximisation du bien-être des travailleurs plutôt que de leurs revenus. Elle se présente comme un problème d'optimisation. En effet, comme les travailleurs décident de la répartition du surplus entre les salaires et le fonds d'investissement et que ce dernier fonds est la seule source possible de financement de l'investissement (autofinancement intégral), on peut établir une aire de possibilités représentant les flux de consommation accessibles. Pour deux périodes, cette aire se dessine dans un plan dont les axes mesurent les niveaux de consommation $C_{1}$ et $C_{2}$. Si l'on suppose ensuite l'existence d'une fonction intertemporelle d'utilité collective sur un horizon de $\mathrm{T}$ périodes, c'est-à-dire $\mathrm{U}=$ $\emptyset\left(C_{1}, C_{2}, \ldots C_{T}\right)$, on trouve le flux optimal de consommation et donc aussi celui de l'investissement en maximisant la fonction $U$ sous la contrainte représentée par l'aire de possibilités. A nouveau, graphiquement et pour deux périodes, cela revient à trouver le point de tangence entre la frontière supérieure de l'aire de possibilités et une courbe d'indifférence intertemporelle aussi élevée que possible.

En fait, l'optimum ainsi atteint peut présenter des caractéristiques fort différentes suivant l'orientation des préférences collectives, le type de fonction de production, la forme et la position de l'aire de possibilités. Cependant, une fois certaines limites du modèle de base abandonnées. Furubotn montre qu'une politique de salaires courants les plus élevés possibles, et donc d'investissements collectifs très faibles, est probable sans être l'unique voie.

A. Dumas et D. Serra (1973) enfin ne font pratiquement que reprendre le modèle de base de Furubotn (1971) mais en spécifiant les formes de la fonction de production et de la fonction d'utilité collective : la première, très simple, se caractérise par des rendements d'échelle constants tandis que la seconde évoque la loi de Fechner par sa forme logarithmique. La résolution du modèle et l'obtention d'une solution optimale pour les flux de consommation et d'investissement deviennent dès lors possibles. Aussi, en scindant l'optimisation dynamique en une suite d'optimisations statiques, les auteurs arrivent à la conclusion suivante : au fur et à mesure que l'on se rapproche de l'horizon temporel, la part du revenu net affecté à l'autofinancement des investissements va en diminuant, celle distribuée sous forme de rémunérations individuel- 
les allant au contraire en augmentant. Si donc, comme le modele le suppose, l'autofinancement représente la seule source financière pour les investissements dans l'entreprise, ceux-ci auront tendance à se réduire au cours du temps.

Les trois analyses dynamiques que nous venons d'examiner font apparaître un sous-investissement lié à l'autofinancement de l'entreprise et tendent donc à confirmer la troisième force de Vanek. Elles sont par contre beaucoup moins intéressantes pour ce qui concerne les autres forces. En effet. les modèles de base de Furubotn ainsi que de Dumas et Serra supposent le facteur travail constant si bien que la première force d'auto-extinction est par hypothèse inexistante et que la force d'inemploi ne peut être testée. Ils intègrent également une hypothèse proche de la réalité yougoslave mais qui empêche la deuxième force d'auto-extinction de se manifester : les travailleurs doivent maintenir la valeur du capital accumulé et, par conséquent, ils ne peuvent en aucun cas désinvestir. Quant au modèle d'Atkinson, il ne dégage pas de résultat significatif en dehors des comportements d'investissement et de prix.

\section{LES F́VAIUATIONS EMPIRIQUES DE I.A THEORIH}

Quelques auteurs ont essayé de tester empiriquement la théorie du financement de l'entreprise autogérée en recherchant dans des expériences concrètes les effets pervers prédits par les modèles.

A. Mc Gregor (1977) pour sa part a cherché les trois premières forces de Vanek (désir de réduire le nombre de membres, tendances à désinvestir et à sous-investir) plus celle qui pousserait les membres à engager des travailleurs sans leur accorder le statut et les droits d'associés. Dans les coopératives produisant du contreplaqué dans le Nord-Ouest des Etats-Unis, qu'il examine à travers les travaux de Bellas (1972) et Berman (1967), il décèle la première force d'auto-extinction sous la forme suivante : certaines coopératives ont prospéré de façon remarquable et le prix de leurs parts de capital est progressivement devenu si élevé qu'il a rendu le sociétariat inaccessible à de nouveaux travailleurs. Par contre. la réduction du nombre de membres s'est nettement moins manifestée dans des coopératives dont la réussite financière a été moindre. C'est pourquoi Mc Gregor parle d'un "paradoxe du succès".

En ce qui concerne la seconde force d'auto-extinction, il relève que trois coopératives prospères furent vendues par leurs membres 
en l'espace d'une génération de travailleurs. Pour la tendance au sous-investissement, il reconnaît que la vérification est assez difficile mais il note qu'entre 1955 et 1965, aucune coopérative n'a créé de nouvelles installations alors que plus de quarante entreprises classiques du même secteur opéraient de tels investissements. Enfin. l'embauche croissante de travailleurs non membres est évidente dans de nombreux cas : le plus frappant est le passage en une trentaine d'années d'une entreprise employant 118 membres et 100 travailleurs non associés à une proportion de 45 membres pour un millier de travailleurs, l'écart entre les rémunérations des deux catégories ayant par ailleurs fortement augmenté.

Mc Gregor retrouve également ces quatre effets pervers dans deux coopératives de plantations de copra en Nouvelle-Guinée, pour lesquelles il a lui-même réalisé des études de cas. Enfin, dans sa thèse de maîtrise (1974), il examine encore la filature de coton de Rochdale, une coopérative anglaise de productions chimiques (la fameuse Scott Bader Company qui sera à la base de l'Industrial Common Ownership Movement) ainsi que l'entreprise autogérée yougoslave. L'ensemble de ces expériences tui paraissent fournir un large support aux thèses de Vanek.

De leur côté, D.C. Jones et D.K. Backus (1977) ont entrepris de tester la théorie du financement d'une manière assez différente. Plutôt que de rechercher les manifestations des quatre forces susdites, ils considèrent l'équilibre de long terme auquel celles-ci sont sensées conduire. En effet, l'analyse de Vanek montre que, comparée à une firme autogérée ou capitaliste en situation de concurrence parfaite et payant pour l'usage du capital un prix reflétant sa rareté, la firme autogérée qui s'autofinance (au sens défini p. 209) va tendre dans le long terme à opérer :

- dans la zone des rendements croissants de la fonction de production,

- à un niveau inférieur de production.

- en sous-investissant et en se caractérisant donc par un rapport capital-travail plus faible.

Jones et Backus effectuent pour chacune de ces prédictions des tests basés sur des données concernant une quinzaine de coopératives anglaises de fabrication de chaussures et couvrant la période 1948-1968. Dans la phase initiale. ils supposent que toutes les coopératives sont intégralement autofinancées. La première prédiction est testée à partir de fonctions de production estimées (CobbDouglas et CES), tandis que les deux autres sont évaluées par comparaisons entre les coopératives et des entreprises capitalistes du même secteur. Cette série d'exercices statistiques offre à la théorie un soutien empirique généralement solide quoique variable. 
Dans une deuxième série de tests, les auteurs différencient les coopératives selon le niveau de participation des travailleurs $\left(P_{j}\right)$ et de propriété collective $\left(\mathrm{ECO}_{\mathrm{j}}\right)$. Ces deux variables sont d'abord introduites dans les fonctions de production comme facteurs additionnels et indépendants. Ensuite d'autres calculs sont effectués pour tester leur action au sein même des fonctions de production (ECO $_{i}$ peut par exemple agir sur le comportement de l'entreprise en limitant l'investissement). Cependant, si les résultats montrent une corrélation notable entre la participation dans l'entreprise et l'efficience de celle-ci, la prise en compte du degré variable de propriété collective et donc d'autofinancement n'apporte guère de confirmation significative aux prédictions théoriques.

Le travail de Jones et Backus a été prolongé par Jones (1980) bien que la vérification de la théorie du financement ne représente plus ici qu'une partie de l'article, consacré dans son ensemble aux effets de différents facteurs sur la productivité de l'entreprise autogérée. Pour notre propos, on retiendra surtout que Jones envisage en plus les coopératives de production du secteur de l'imprimerie et fonde son étude sur deux fonctions de production de type CobbDouglas. Le principal résultat est le soutien important que reçoit, par le biais des coefficients d'échelle estimés, l'hypothèse d'une échelle de production inefficiente. Mais la confirmation de la théorie est à nouveau moins forte lorsque est examiné l'impact des facteurs $\mathrm{ECO}_{i}$ et Age (les vieilles coopératives sont sensées être plus sensibles a la force de sous-investissement). Néanmoins, ces deux dernières variables sont dans tous les cas négativement, quoique faiblement, correlées à la productivité dans les imprimeries coopératives.

Sans procéder à une véritable étude empirique, H.G. Nutzinger (1975) affirme que l'échec final des coopératives de production anglaises et allemandes, et dans une moindre mesure des coopératives françaises, peut s'analyser à partir du problème de l'autofinancement. En effet, dit-il, ces coopératives s'autofinançaient dans une large mesure et, malgré la rareté des recherches systématiques sur leur évolution, les informations que l'on possède confirment presque toutes les conclusions de la théorie. C'est particulièrement le cas des observations faites par $\mathrm{H}$. Witelsberger (1969) pour les coopératives allemandes.

Pour J. Vanek lui-même (1976), l'économie yougoslave représente une illustration de certains effets prédits par son modèle. De fait, on sait qu'en Yougoslavie les travailleurs des entreprises autogérées ne récupèrent à aucun moment leurs parts dans l'accumulation collective de capital. Les entreprises quant à elles ne doivent pas payer, pour l'ensemble du capital qu'elles utilisent, un intérêt reflétant la rareté de celui-ci. Certaines sommes sont bien versées au titre d'intérêts sur des fonds empruntés ou au titre de taxes sur 
le capital mais, au vu d'une inflation particulièrement forte, elles ne représentent pas grand-chose. Les revenus du capital sont donc pour l'essentiel des rentes qui échoient aux travailleurs. Or, en s'appuyant surtout sur les travaux de A. Vahcic (1976), Vanek constate que, si les performances de l'économie yougoslave sur les plans de la croissance et de l'accumulation sont respectivement semblables et supérieures à celles de nombreux pays capitalistes, elles sont par contre très pauvres en ce qui concerne la création d'emplois dans le secteur moderne (industrie et services). Autrement dit, les entreprises modernes sont très capitalistiques mais n'atteignent pas la taille efficiente correspondant à leurs stocks du capital. Aussi peut-on les situer sur le lieu aa du graphique 3 (dans la zone des niveaux de production inefficients) mais nettement à gauche et au-dessus du point a ${ }^{*}$ car, selon Vanek, la force de sous-investissement est compensée par certains facteurs : notamment les pressions morales et politiques exercées du dehors de l'entreprise ainsi que les contrats sociaux visant à équilibrer les revenus individuels des travailleurs et les sommes retenues pour l'accumulation.

Sans prétendre trouver là une relation de cause à effet, on peut encore noter que le complexe de Mondragon, où l'autofinancement irrécupérable et non rémunéré est faible, constitue précisément un exemple remarquable de réussite économique, comme en témoigne le tout récent ouvrage de H. Thomas et C. Logan (1982) (12).

Finalement, dans un sens tout différent, K.V. Berman (1980) prétend que l'évolution des coopératives américaines fabriquant du contreplaqué a été faussement interprétée en faveur de certaines prédictions théoriques parmi lesquelles celles de Vanek sur l'autofinancement. Ainsi, estime-t-elle, si certaines coopératives ont réduit le nombre de leurs membres, c'est pour des raisons très différentes de celles avancées par Vanek et Mc Gregor : notamment la nécessité de s'adapter à une technologie "labor-saving" ainsi que la perspective des multiples problèmes qu'aurait engendrés l'appel de nouveaux membres. Elle reconnaît également qu'un nombre assez élevé d'entreprises ont été vendues par leurs membres mais, ditelle, ces ventes étaient généralement motivées par des exigences financières. Seules trois de ces coopératives étaient prospères. Enfin, le sous-investissement a certes constitué un problème crucial mais pas conformément à la théorie. En effet, comme l'explique

(12) Ce livre fournit des analyses détaillées tant de la structure financière que de la performance économique de ces coopératives du pays basque espagnol. A. Gutierrez-Johnson (1978), de son côté, relève qu'en 1975, le capital d'une nouvelle coopérative de Mondragon provenait en moyenne pour $80 \%$ de fonds extérieurs. prêtés surtout par la Caja Laboral Popular, et qu'une proportion des $20 \%$ restants consistait en des parts individuelles de capital. récupérables et rémunérées. 
C.E. Gunn (1980), les revenus mis en réserve par les coopératives du contreplaqué sont considérés comme des revenus appartenant aux membres et sont d'ailleurs crédités aux comptes individuels de ceux-ci. Bien que non rémunérés en tant que tels, ces capitaux sont donc récupérables, ce qui signifie pour Berman que le raisonnement à la base de la troisième force ne peut s'appliquer ici de la même manière. Pour elle, c'est en réalité l'incapacité des associés à comprendre la nécessité d'investir à long terme, qui a amené de graves problèmes de sous-investissement.

\section{CONCLUSIONS}

Au terme de cette synthèse sur l'autofinancement de l'entreprise autogérée, résumons les principaux enseignements de la littérature passée en revue, ses limites ainsi que quelques perspectives.

I. Le modèle de base de Vanek avec ses quatre forces négatives a été largement enrichi par un certain nombre de contributions théoriques qui ont rendu l'analyse plus réaliste. Il importe néanmoins de ne pas perdre de vue les limites de ces approches formalisées.

D'une part les résultats auxquels ces dernières aboutissent peuvent être très dépendants de certaines hypothèses, parfois même apparemment sans grande importance. Ainsi pour N. Zafiris (1982), l'hypothèse d'une durée de vie illimitée du capital et par là l'exclusion des problèmes de dépréciation et d'amortissement conduit Vanek (1971) à une interprétation erronée du problème du sousinvestissement $\left({ }^{13}\right)$. F.H. Stephen (1981) de son côté soutient que le capital utilisé par les travailleurs, même s'il provient d'un autofinancement, a un coût d'opportunité, et que c'est l'omission de ce coût dans l'analyse de Vanek qui est responsable des principaux résultats du modèle $\left({ }^{14}\right)$.

D'autre part, il ne faut pas non plus oublier que les apports théoriques repris ici s'inscrivent fondamentalement dans une même ligne et dans le cadre de certaines hypothèses communes. D'autres voies mériteraient d'être explorées à partir de modèles construits

(1.3) Zafiris estime que le véritable "disincentive "a l'investissement n'est pas tant le caractère non récupérable des investissements materiels que l'obligation souvent imposée aux coopératives de maintenir. au moins dans une large mesure, la valeur de leur capital.

(14) La prise en considération de ce coût d'opportunité montre. selon Stephen, que l'autofinancement va seulement engendrer une préférence plus forte pour des modes de production laboristiques 
sur des bases toutes différentes. Par exemple, on a vu qu'Atkinson (1973) étudie ce qu'il nomme une "managerial labor-owned firm ". Pour cette demière, il trouve qu'un accroissement de l'autofinancement aura comme effet probable d'accélérer la croissance de l'entreprise. Une vision alternative de la firme autogérée a aussi été proposée par Furubotn (1976) pour qui l'objectif poursuivi est le bien-être du groupe des travailleurs qui ont pu former une majorité dans l'entreprise, avec tout ce que cela comporte comme manœuvres pour garder le contrôle de celle-ci. M. Jensen et $\mathbf{W}$. Meckling (1979) quant à eux redéfinissent la fonction de production pour y incorporer sa dépendance vis-à-vis de la structure des droits de propriété dans une économie d'autogestion et vis-à-vis des droits contractuels dans le cadre desquels l'entreprise opère. D'autres auteurs pourraient encore être cités mais, dans ces approches alternatives, le problème de l'autofinancement n'a généralement pas encore constitué une préoccupation essentielle.

2. Malgré les lacunes des modèles envisagés, les effets pervers de l'autofinancement mis par eux en évidence doivent retenir notre attention. Ils ont effectivement reçu d'études de cas minutieuses et de tests statistiques élaborés une certaine confirmation empirique. D'importants efforts supplémentaires s'avèrent cependant nécessaires dans ce domaine car, pour l'autogestion comme pour les autres grands thèmes économiques, l'inspiration théorique de nombreux chercheurs n'a d'égale que leur aversion pour les ingrates démarches empiriques.

De plus, même lorsque les faits recoupent certaines prédictions de la théorie, la prudence s'impose pour la validation de celle-ci. Comme le fait remarquer Berman (1980), découvrir par exemple un sous-investissement n'équivaut pas à déceler la raison de ce comportement.

Dans le cadre d'une large étude des SCOP françaises, nous avons récolté des séries statistiques originales couvrant des périodes de 10 à 20 ans, et ce pour près de 400 coopératives. A condition de pouvoir surmonter certaines difficultés dans la mesure de l'autofinancement de ces entreprises, nous espérons apporter bientôt une contribution supplémentaire à la vérification de la théorie.

3. Si, comme on vient de l'admettre, l'autofinancement des entreprises autogérées peut engendrer effectivement certains problèmes, la création d'organismes financiers adaptés à ce type d'entreprises ne peut qu'être encouragée. De fait, bien que ces problèmes ne se posent sans doute pas d'une façon aussi cruciale que le pense Vanek, la mise sur pied de fonds comme celui envisagé au niveau de la région wallonne tire de la théorie économique une certaine justification puisque ceux-ci peuvent contribuer à limiter le financement interne des coopératives de travailleurs. 
4. En reconnaissant l'utilité voire la nécessité de sources de financement externe, on n'a encore rien dit des conditions dans lesquelles celui-ci doit idéalement s'opérer. Car c'est bien relever un défi que de vouloir concilier le droit des travailleurs de gérer eux-mêmes leur entreprise et certaines exigences légitimes des pourvoyeurs privés ou publics de capitaux. En plus d'un large débat sur le cas particulier de la Yougoslavie, ce thème a suscité diverses idées chez un certain nombre d'auteurs parmi lesquels H.G. Nutzinger (1975), J. Vanek (1977 et 1979), R.A. McCain (1977), A. McGregor (1977), E. Schlicht et C.C. von Weizsäcker (1977), E.G. Furubotn (1980) et O. Chillemi (1981). Un "survey " de ces contributions pourrait être l'objectif d'un prochain travail.

Enfin, il importe de souligner que, pour les théories étudiées dans notre troisième chapitre, l'autofinancement n'engendre certains "effets pervers" que si les fonds investis sont, par leur nature collective, irrécupérables et si la rente provenant de ces capitaux n'est pas extraite par le paiement d'un prix reflétant la rareté de ceux-ci. Autrement dit, un financement interne sur base de participations individuelles rémunérées et récupérables se révèle tout aussi souhaitable qu'un financement externe. Une telle formule a notamment été développée avec beaucoup de succès dans les coopératives de Mondragon et constitue à notre avis une solution quasiment idéale. Car, en outre, l'expérience prouve que la motivation de travailleurs impliqués dans le capital de leur entreprise est bien différente de celle de simples employés. Cela ne signifie pourtant pas qu'en vue d'une réelle autogestion, la qualité de coopérateur ne doive être attachée au travail, c'est-à-dire à l'" apport en industrie " plutôt qu'au capital.

\author{
BIBI.IOGRAPHIF.
}

ATKINSON A.B. (1973), "Worker Management and the Modern Industrial Enterprise". Quarterly Journal of Economics, Vol. 87, No. 3, pp. 355-392.

Bell.AS C. (1972), Industrial Democracy and the Worker-Owned Firm, New York, Praeger.

Berman K.V. (1967), Worker Owned Plywood Companies : An Economir Analysis, Washington State University Press, Pullman.

BERMAN K.V. (1980), Comparative Efficiency under Worker Management : Some Considerations from Labor-Management Theory in Relation to U.S. Plynood Cooperative Experience, Hamilton College, Paper presented at the Symposium on Economic Performance of Participatory Firms.

Chil. emI O. (198I). "Sul regime del capitale nell'impresa autogestita ". Rivista Internazionale di Scienze Economiche e' Commerciali, No. 1-2, pp. 151-169 
DAURES N. \& Dumas A. (1977), Théorie économique de l'autogestion dans l'entreprise, Montpellier, Editions du Faubourg.

DEFOURNY J. (1982), Les nouvelles coopératives, espoir pour la Wallonie?, Liège, CIRIEC, Working Paper No. 82/01.

DOMAR E.D. (1966), "The Soviet Collective Farm as a Producer Cooperative", American Economic Review, vol. 56, pp. 734-757.

Dumas A. (1981), ed., L'autogestion, un système économique?, Paris, Dunod.

Dumas A. \& Serra D. (1973), "Modèle d'autofinancement dans l'entreprise autogérée ", Revue d'économie politique. No. 6, pp. 1022-1041.

Furubotn E.G. (1971), "Toward a Dynamic Model of the Yugoslav Firm ", Canadian Journal of Economics, No, 4, pp. 182-197.

FurubotN E.G. (1976), "The Long-Run Analysis of the Labor-Managed-Firm : An Alternative Interpretation". American Economic Review, vol, 66, pp. 104-123.

FuRUBOTN E.G. (1980), "Tradable Claims and Self-Financed Investment in the Capitalist Labor-Managed Firm ", Zeitschrift für die Gesamte Staatswissenschaft, No. 4.

Furubotn E.G. \& PEJovich S. (1970), "Property Rights and the Behavior of the Firm in a Socialist State : The Example of Yugoslavia ", Zeitschrift für Nationalökonomie, Vol. 30 (3-4), pp. 431-454.

GunN C.E. (1980), "Plywood Cooperatives of the Pacific Northwest : Lessons for Workers' Self-Management, Vol. 14, No, 3, pp. 393-416.

GutTierrez-Johnson A. (1978), «Compensation, Equity and Industrial Democracy in the Mondragon Cooperatives ", Economic Analysis and Workers' Management, Vol. 12, No. 3-4, pp. 267-290.

Jfensen M. \& Mackling W. (1979), « Rights and Production Functions : An Application to Labor-Managed Firms and Codetermination", Journal of Business, Vol. 52. No. 4, pp. 469-506.

Jones D.C. (1980), Productivity and British Producer Cooperatives, Hamilton College, Paper presented at the Symposium on Economic Performance of Participatory Firms.

Jonfs D.C. \& Backus D.K. (1977). "British Producer Cooperatives in the Footwear Industry : An Empirical Evaluation of the Theory of Financing ". The Economic Journal, Vol. 87. No. 347, pp. 488-510.

MCCAIN R.A. (1977), "On the Optimum Financial Environment for Worker Cooperatives", Zeitschrift für Nationalökonomie. Vol, 37. No. 3-4, pp. 355-384.

MCGREGor A. (1974), Capital Rent Extraction and the Survival of the Producer Cooperative, Cornell University, Ithaca (N.Y.). Program on Participation and Labor-Maraged Systems. Monograph No. 9.

MCGregor A. (1977), "Rent Extraction and the Survival of the Agricultural Production Cooperative", American Journal of Agricultural Economics, Vol. 59. No. 3, pp. 478-488.

NuTZINGER H.G. (1975), « Investment and Financing in a Labor-Managed Firm and its Social Implications ", Economic Analysis and Worker' Management, No. 3-4.

Pejovich S. (1969), "The Firm, Monetary Policy and Property Rights in a Planned Economy ». Western Economic Journal, Vol. 7. pp. 193-200.

Pfistieal: P. \& Quaden G. (1978), «Autogestion et coopération ", Annales de l'économie publique, sociale et coopérative, No. 2, pp. 81-84.

SaIvf M.-A. (1981), "L'Etat et le développement coopératif », Annales de l'économie publique, sociale et coopérative, No. 3, pp. 301-315.

SCHI.ICHT F. \& von WEIZSÄKER C.C. (1977), "Risk Financing in Labour-Managed Economies : The Commitment Problem". Zeitschrift für die Gesamte Staatwissenschaft. Special Issue on Profit Sharing. 
SifpHF.N F.H. (1981), On the Analysis of the Investment Behaviour of Producers Cooperatices .. Glasgow, University of Strathclyde. Strathclyde Discussion Papers in Economics, No. 81/5.

Thomas H. \& IKGAN C. (1982). Mondragon : an Economic Analysis, London, Allen and Unwin.

VAHCIC A. (1976). An Ecunometric Analysis of Post War Performance of Yugoslas Economy, Cornell University. Ithaca (N.Y), Series of Unpublished Studies. No. 13, Program on Participation and Labor-Managed Systems.

VANFK J. (1970). The General Theory of Labor-Managed Market Economic's. Comell University Press, Ithaca (N.Y.).

Vanf: J. (1971). The Basic Theory of Financing of Participaton Firms, Cornell University Department of Economics, Ithaca (N.Y.). Working Paper No. 27. Reprinted in Vanek (1975) and Vanek (1977).

VANEK J. (1975), ed,, Self-Management : Economic Liberation of Man. Harmondworth, Penguin.

VANEK J. (1976), "Self-Management. Workers' Management, and Labor Management in Theory and Practice : a Comparative Study ". Economic Analysis and Workers' Management. No. 1. pp. 5-24.

VANEK J. (1977). The Labor-Managed Economy : Essays. Ithaca (N.Y.). Comell University Press.

VANEK J. (1979), A System for Worker Participation and Self-Management in Western Industrialized Economies. Glasgow. Paper presented at the Walton Symposium on Self-Management. 
Copyright of Annals of Public \& Co-operative Economy is the property of Blackwell Publishing Limited and its content may not be copied or emailed to multiple sites or posted to a listserv without the copyright holder's express written permission. However, users may print, download, or email articles for individual use. 